\title{
Voces reconocidas en Comunicación Organizacional en de América Latina'
}

\section{Vozes reconhecidas na Comunicação Organizacional na América Latina}

\section{Recognized voices in Organizational Communication in Latin America}

a Comunicación Organizacional en América Latina, tiene hoy un desarrollo importante, gran parte gracias al trabajo que han logrado conjuntar dos mujeres: María Antonieta Rebeil Corella, desde México, y Margarida Krohling Kunsch, desde Brasil. Ambas con visiones que conjugan esfuerzos para impulsar el crecimiento de la Comunicación Organizacional considerando el trabajo previo de Europa y Estados Unidos, pero enfocándolo en nuestros contextos Latinoamericanos.

La entrevista se desarrolla en el encuentro Escuchando nuestras voces: perspectivas latinoamericanas en Comunicación Organizacional, en el que más de 30 académicos e investigadores se reúnen para dialogar con las contribuciones teóricas de investigación y las distintas denominaciones a la Comunicación Organizacional que se le atribuyen en nuestros países latinoamericanos. En este escenario, María Antonieta y Margarida nos comparten su experiencia y visión como líderes en el desarrollo de la Comunicación Organizacional. Este conversatorio es un ejemplar único en el que se encontrará la respuesta de líderes latinoamericanas respecto a la Comunicación Organizacional. 


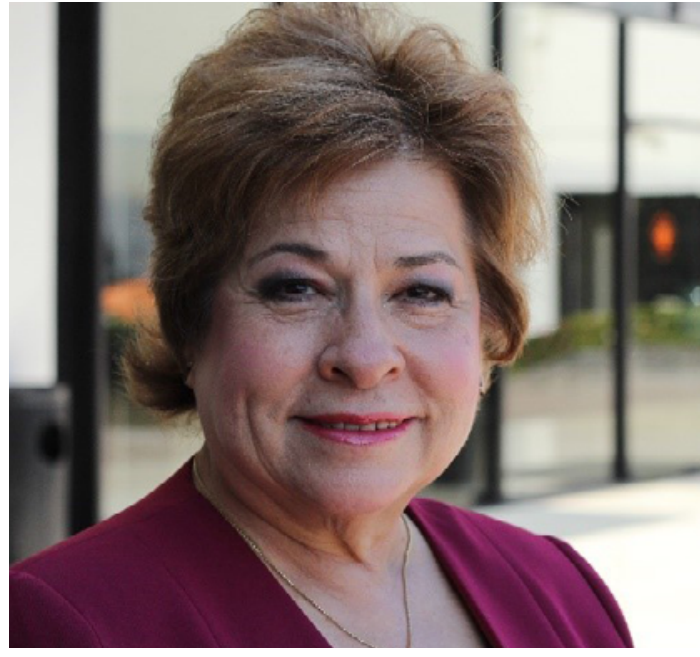

Foto: María Antonieta Rebeil Corellas

\section{María Antonieta Rebeil Corella}

Doctora en Ciencias Sociales con especialidad en Sociología Organizacional por la Universidad Iberoamericana

Universidad Anáhuac, México

Maestría en Educación y Desarrollo por la Universidad de Stanford Licenciatura en Ciencias de la Comunicación por el Iteso de Guadalajara

- $\quad$ Es miembro del Sistema Nacional de Investigadores Nivel 2 del Consejo de Ciencia y Tecnología.

- Fundadora del Centro de Investigación para la Comunicación Aplicada (Cica) de la Facultad de Comunicación de la Universidad Anáhuac México. Ella ha sido la directora desde el 2014 a la fecha. Fundó del programa Doctoral en Comunicación Aplicada y el Doctorado en Investigación de la Comunicación que opera desde 2010 hasta la fecha.

- Coordinadora ejecutiva de la Red Internacional en Investigación y Consultoría en Comunicación (Riicc)

- Coordinadora en Grupo de Trabajo 2 de Comunicación Organizacional y Relaciones Públicas de Alaic

- Coordinadora de Investigación delConsejo Nacional para la Enseñanza e Investigación de las Ciencias de la Comunicación (Coneicc)

- Vicecoordinadora del Grupo de Investigación de Comunicación Organizacional en la Asociación Iberoamericana de Comunicación (Asibercom)

- Coautora de más de 15 libros y números artículos de revista arbitradas y de especialidad a nivel nacional e internacional

- E-mail: arebeil@anahuac.mx 


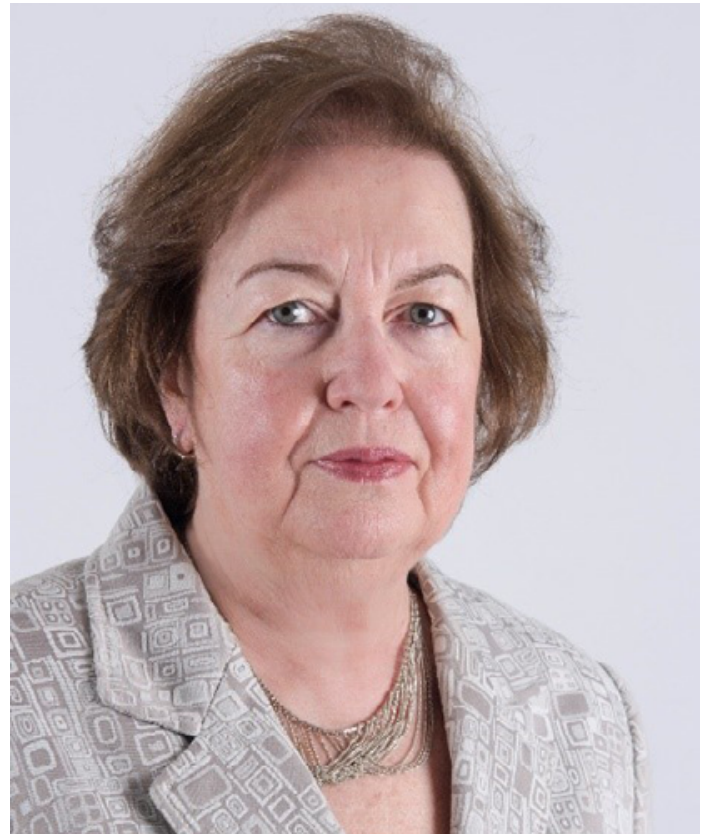

Foto: Margarida Krohling Kunsch

\section{Margarida Krohling Kunsch}

- $\quad$ Doutora em Ciências da Comunicação e livre-docente em Teorias e Processos de Comunicação Institucional, pela Escola de Comunicações e Artes da Universidade de São Paulo (ECA-USP)

- Professora titular e ex-diretora da Escola de Comunicações e Artes de Universidade de São Paulo.

- Pró-Reitora Adjunta da Pró-Reitoria de Cultura e Extensão da Universidade de São Paulo

- $\quad$ Pesquisadora vinculada ao Conselho Nacional de Desenvolvimento Científico e Tecnológico (CNPq), do Ministério de Ciência e Tecnologia do Brasil.

Autora de vasta produção científica em Ciências da Comunicação e em Comunicação Organizacional e Relações Públicas. Publicou três livros próprios, com destaque para Planejamento de relações públicas na comunicação integrada, 89 capítulos de livros e 32 artigos em periódicos científicos nacionais e internacionais e organizou 40 coletâneas dessas áreas.

- Coordenadora do Centro de Estudos de Comunicação Organizacional e Relações Públicas (Cecorp) da ECA-USP.

- $\quad$ Presidente da Associação Brasileira de Pesquisadores de Comunicação Organizacional (Abrapcorp) e de Relações Públicas e do Conselho Curador da Sociedade Brasileira de Estudos Interdisciplinares da Comunicação (Intercom).

- Foi Presidente da Sociedade Brasileira de Estudos Interdisciplinares da Comunicação (Intercom), Federação Brasileira das Associações Científicas e Acadêmicas de Comunicação (Socicom), Asociación Latinoamericana de Investigadores de la Comunicación (Alaic), Confederação Ibero-Americana das Associações Científicas e Acadêmicas de Comunicação (Confibercom)

- $\quad$ Criadora, diretora responsável e editora de duas revistas científicas: Organicom - Revista Brasileira de Comunicação Organizacional e Relações Públicas, da ECA-USP; e Revista Latinoamericana de Ciências de la Comunicación, da Asociación Latinoamericana de Investigadores de la Comunicación (Alaic).

- E-mail:mkkunsch@usp.bre mkkunsch@uol.com.br 
Griselda - Buenas tardes, gracias por aceptar la invitación. En este Conversatorio "Voces reconocidas de América Latina" invitamos a dos mujeres reconocidas en América Latina por su trayectoria en el ámbito de la Comunicación Organizacional. La doctora Margarida Krohling Kunsch de la Universidad de São Paulo, Brasil, y la doctora María Antonieta Rebeil Corella de la Universidad Anáhuac, México. Los aportes de ambas han sido cruciales en la configuración del campo en nuestra región.

Iniciamos con algunas preguntas que actualmente nos hacemos los académicos, investigadores y profesionales buscando certeza en este campo de la Comunicación Organizacional. ¿Cuáles son las bases para conceptualizar la Comunicación Organizacional?, ¿qué relación ha mantenido esa conceptualización con la Escuela Latinoamericana de Comunicación(ELACOM)?, ¿desde dónde conceptualizamos, a partir de lo que estamos viviendo, nuestras realidades sociales? y ¿cómo abordarlo?

María Antonieta - Bueno, en primer lugar, muchas gracias, es un honor para mí que me hayan considerado en este conversatorio con tan distinguidas colegas, Margarida y Griselda. En segundo lugar, yo quiero empezar por la parte de ¿cuál ha sido la influencia de la Escuela Latinoamericana de la Comunicación en esta concepción que tenemos de la Comunicación Organizacional en América Latina? La Escuela Latinoamericana presenta, de manera muy sencilla, para nosotros, la base en la cual fuimos formados como comunicólogos con el pensamiento del doctor Marques de Melo, quien lamentablemente se nos ha adelantado y que nos tiene a todos aun tristes con esa enorme pérdida para el campo de la Comunicación. José Marques y su grupo de Asociación Latinoamericana de Investigadores en Comunicación (ALAIC) fueron los primeros pensadores que se atrevieron a decir: "sí, tenemos una Escuela distinta, distinta a la de Frankfurt, a la de Inglaterra, a la de Estados Unidos, tenemos algo muy diferente que expresar sobre la disciplina de la Comunicación, y tomamos el reto de sistematizar". El doctor Marques de Melo y otros colegas que le fueron siguiendo, como es el caso del Dr. Gustavo Adolfo León Duarte de la Universidad de Sonora, han continuado esa tradición tratando de sistematizar esta Escuela Latinoamericana de la Comunicación. En este marco, como latinoamericanos fuimos formados, no sin voltear a las otras influencias que teníamos desde Europa y desde Estados Unidos.

Cabe señalar que se dice que una escuela del pensamiento se conforma o se institucionaliza a partir de tres factores: el primero es que existan los programas educativos en las universidades para formar a profesionales, en este caso, de la Comunicación y, muy particularmente en el marco del interés de los aquí presentes, a comunicadores organizacionales; segundo, que existan revistas científicas de comunicación, cabe señalar que en América Latina la revista ORGANICOM fue fundada y sigue vigente gracias al esfuerzo y visión de Margarida Khroling Kunsch; y tercero, que existan asociaciones como ALAIC, Asociación Iberoamericana de Comunicación (ASIBERCOM) y ahora, en nuestro caso de comunicadores organizacionales, la Asociación Mexicana de Investigadores y Profesionales de la Comunicación Organizacional (AMIPCO), que hoy nos convoca y que por cierto es algo que Griselda Guillén muy hábilmente ha realizado y continúa haciendo. Yo diría que esos tres factores están muy avanzados y bastante maduros en nuestro contexto. Considero que esto ha sido la gran influencia de la Escuela Latinoamericana para definir quiénes somos en este momento.

Margarida - Muchas gracias. Es un placer estar ahora aquí. Felicitaciones por la organización perfecta del evento, que considero que es un encuentro que va a generar buenos frutos. Con relación a la pregunta sobre la Escuela Latinoamericana, no quiero repetir lo que Antonieta ya manifestó. Quiero destacar también el papel de ALAIC y el grupo de trabajo en Comunicación Organizacional y Relaciones Públicas, que fue la forma que encontramos para reunir un poco el pensamiento comunicacional latinoamericano de estas áreas.

Un poco con relación a la pregunta ¿cuáles son las bases de la conceptualización de comunicación organizacional? La Comunicación Organizacional tiene sus raíces en varios campos, como los estudios organizacionales, la administración y las teorías de las organizaciones; la sociología y la psicología social y organizacional; la antropología, la lingüística y la retórica; 
y la teoría de la comunicación. Por tanto, sus estudios involucran varias ciencias en una perspectiva interdisciplinar. Los estudiosos de esas áreas fueron los primeros en desarrollar trabajos que señalan preocupación por la comunicación en las organizaciones y dieron la posibilidad de ser vistos como los precursores de un campo que hoy es considerado como una disciplina académica. A diferencia del pasado, donde las bases estaban en la psicología social y la sociología, hoy tenemos teorías propias más centradas en las Ciencias de la Comunicación. Yo creo que en la Comunicación Organizacional en América Latina tenemos grandes avances y hemos defendido una visión amplia, abarcadora y holística. En la obra colectiva Comunicação organizacional, organizada por mí en 2009, en dos volúmenes, los autores abordan diversas percepciones teóricas y aplicadas.

Entiendo la Comunicación Organizacional primero como un fenómeno comunicacional dentro de las organizaciones y todo su contexto político, económico y social; además, existe un fenómeno inherente a la naturaleza de las organizaciones y a los grupos de personas que la integran; la Comunicación Organizacional envuelve los procesos comunicativos y todos sus elementos constitutivos. En ese contexto, se hace necesario ver a la comunicación insertada en procesos simbólicos y enfocada en los significados de los agentes involucrados, de los relacionamientos interpersonales y grupales, valorizando las prácticas comunicativas cotidianas y las interacciones en sus formas más diversas de manifestación y construcción social. Por tanto, en esa línea de pensamiento se supera la visión lineal e instrumental de la comunicación por una visión mucho más compleja y abarcadora.

Otra vertiente para considerar es el alcance de la Comunicación Organizacional, en la cual se configuran las diferentes modalidades que permean su concepción y sus prácticas. Es lo que, ya desde los años 1980, de forma pionera, denomino "Comunicación Organizacional Integrada", comprendiendo la comunicación institucional, la comunicación mercadológica, la comunicación interna y la comunicación administrativa, que acontece a partir de objetivos y propósitos específicos. Bajo esta perspectiva, las acciones comunicativas necesitan ser guiadas por una filosofía y una política de comunicación integrada que tengan en cuenta las demandas, los intereses y las expectativas de los públicos/sujetos involucrados y de la sociedad. Debe haber total integración entre esas modalidades comunicacionales para la búsqueda y el alcance de la eficacia, la eficiencia y la efectividad organizacional en beneficio de los públicos y de la sociedad como un todo, y no solo de las organizaciones.

Como se puede observar, la Comunicación Organizacional, en esta perspectiva global, es por sí sola compleja. En ese sentido, el área de la comunicación deja de tener una función apenas táctica y pasa a ser considerada estratégica. 0 sea, debe considerar la cuestión humana y agregar valor a las organizaciones, es decir, debe ayudar a las organizaciones a valorar a las personas y a cumplir su misión, alcanzar sus objetivos globales, contribuir en la fijación pública de sus valores y en las acciones para alcanzar su ideario en el contexto de una visión de mundo bajo la égida de los principios éticos. Si consideramos la perspectiva crítica como una de las características del pensamiento comunicacional latinoamericano, podemos defender una visión mucho más amplia de la Comunicación Organizacional y no restringida a una concepción instrumental y funcionalista, e identificar de alguna forma esa relación.

Muchos estudios que vienen siendo desarrollados en Brasil, sobre todo a nivel de postgrado, han tenido una preocupación en trabajar en la línea crítica y según la teoría de la complejidad. Sin embargo, de acuerdo con mi experiencia como investigadora y orientadora de tesis, no se ha dado un énfasis a la vinculación de la Comunicación Organizacional y de las Relaciones Públicas a la ELACOM.

Griselda - Respecto a los abordajes teóricos y metodológicos de la comunicación organizacional. Desde su punto de vista y su trayectoria, ¿cómo ha evolucionado el campo, ¿qué han observado, incluso ustedes que han vivido, propuesto y desarrollado aspectos teóricos y metodológicos? 
María Antonieta - Yo diría que la Comunicación Organizacional ha presentado grandes retos tanto teóricos como metodológicos. En primer lugar, muchas personas consideran que la misma comunicación no es una disciplina en sí. Esta es la primera batalla que tenemos que librar. Nos insisten que se trata de nada más que un campo de estudio en donde confluyen la Antropología, la Sociología, las Ciencias Políticas, la Psicología Social, la Cibernética, la Semiología, la Lingüística, entre otras. Al volver la vista hacia quienes nos decimos comunicólogos, con frecuencia suelen considerarnos creadores de posters, que podemos combinar colores divinamente o que somos muy hábiles para comunicar bien o de manera accesible lo que otros piensan. La Comunicación en sí es una disciplina y tiene mucho que aportar a la sociedad, es lo que permite que se cambie, que fluya y que se transforme en algo cada vez mejor. Esa, es la primera batalla, lo siguiente es ¿cómo entender esta subdisciplina que es la Comunicación Organizacional? Empiezan a fluir una serie de ideas, pasamos de un concepto muy administrativo, muy gerencial, y se ha ido complejizando y hasta el día de hoy, uno de los colegas que estaba en una de las mesas en este evento, dio en el clavo, Curvello de Brasil, nos dijo de manera natural, "nos están invitando a los Comunicólogos Organizacionales a concebir a las organizaciones, instituciones, asociaciones y comunidades, porque, finalmente, todas estas son formas organizativas"; y algo mucho más amplio que la comunicación es cómo concebir esta serie de núcleos alrededor de los cuales se organiza la sociedad más compleja. Creo que este reto de cómo concebimos la organización y cómo la comunicación da vida a esta concepción y la hace posible. Considero que es al mismo tiempo un gran reto, pero también una maravilla creada por el hombre, en la que hay que ir reflexionando.

Margarida - Creo que en América Latina hubo grandes avances, pero aún faltan bases más consistentes de teorías del campo y de referenciales metodológicos más específicos dirigidos a esta área. Tenemos pocos estudios sobre teorías de la comunicación en general. Una perspectiva que me parece importante destacar de nuestra realidad brasileña es que trabajamos mucho la perspectiva crítica. Es una característica también de la ELACOM. Con relación a la metodología, podemos considerar las varias vertientes que han contemplado la producción científica disponible, sobre todo de las disertaciones de maestría y de las tesis de doctorado del campo de la Comunicación Organizacional y de las Relaciones Públicas en el país. Se identifican diferentes métodos y procedimientos metodológicos utilizados por los autores. También ocurre que algunas veces las bases del referencial metodológico empleadas no siempre están claras en las producciones científicas disponibles. Defendemos la necesidad y la importancia de la investigación empírica, considerando que nosotros somos parte de las ciencias sociales aplicadas. Las Ciencias de la Comunicación son parte de las ciencias sociales.

Entonces, la pregunta es ¿cómo se construye una teoría? Es justamente a partir de los estudios de las prácticas que reunimos un conjunto de datos que posibilitan traer nuevas reflexiones y construir teorías. Necesitamos incentivar más, lo hacemos sí, pero hay que buscar aportes financieros porque cuesta caro hacer investigaciones empíricas enfocadas en fenómenos y ciertas realidades observables por parte de un investigador. Mi experiencia también, como investigadora y orientadora, es que son varias las dificultades que hemos encontrado para tener acceso, para que las organizaciones colaboren, no hay una cultura científica para eso. Lo veo como un desafío para que la gente cree más estudios. Otra cosa importante que debo destacar es que la teoría y la práctica no deben estar separadas. Una buena teoría debe dar la base para una investigación empírica. Por eso es muy importante mapear, conocer la producción científica que vienen generando los países.

Siento también que es el momento para tener estudios comparativos, Luiz Alberto de Farias desarrolló, a nivel de doctorado, con el apoyo de María Antonieta, un estudio comparativo entre los campos académicos de Comunicación Organizacional y Relaciones Públicas en Brasil y México. Considero que necesitamos conocer las características de la producción de cada país para tener una visión más amplia, de conjunto. Otro aspecto para considerar es que la literatura sobre metodología en comunicación está centrada sobre todo en las ciencias sociales y en innumerables manuales de metodología científica general. Creo que ésta necesita ser revitalizada y ampliada con adaptaciones más pertinentes para los diferentes campos de la Comunicación, sobre todo en la aplicación a los campos de la Comunicación Organizacional y de las Relaciones Públicas. 
Griselda - Bien y con respecto a este desarrollo de la Comunicación Organizacional desde el punto de vista teórico y metodológico, consideran que esto que se ha desarrollado en Latinoamérica ¿aporta algo a las ciencias sociales, hemos aportado cosas distintas o todavía estamos debiendo en ese aspecto?

María Antonieta - Creo que lo que hemos hecho es complejizar el fenómeno mismo, lo hemos hecho más sofisticado, con el apoyo de muchos colegas. Por ejemplo, aquí entre nosotros está Antonio Castillo quien ya empezó a hablar del lobbying, un tema que anteriormente no solíamos incluir en el campo de la Comunicación Organizacional. Están los temas de la crisis, de los conflictos que trabajamos de una manera particular, pues en nuestro país trabajamos crisis de todo tipo, desde las naturales hasta las que creamos nosotros como sociedad. Y ahí también hemos ido aportando. También voy a referirme a Eva María Pérez, quien también está presente. Eva hizo un trabajo exhaustivo de cómo la ética tiene que estar incluida en los estudios de la comunicación, y cómo sin ese componente fundamental no estamos siendo auténticos comunicólogos organizacionales. En el público está Cristina Barroso quien nos ha señalado, de manera muy destacada, acerca de los temas de la interculturalidad, conflictos y oportunidades que se dan cuando dos organizaciones se encuentran para trabajar en conjunto. Incluso, a veces se trata de organizaciones que hablan el mismo idioma. Hay tanto que decir sobre la cultura organizacional que apenas hemos iniciado su análisis. También, Rebeca Arévalo presente nos ha invitado a sumar el tema de la confianza, del capital social, así como en las tramas del liderazgo en las empresas. En este encuentro de estudiosos iberoamericanos que hoy alberga la Universidad de Costa Rica, estamos reunidos tantos que han contribuido de manera fundamental a que este fenómeno se estudie de manera más compleja. Hoy se nos marcan nuevas pautas, nuevos retos para la evolución de esta ciencia. Este continente, junto con España, hemos aportado nuevas vertientes de acercamiento al fenómeno. En Estados Unidos, los estudiosos permanecieron muchos años en los enfoques Psicosociales y de la Administración como fuente de explicación de la Comunicación Organizacional. Ciertamente, uno de los puntos de quiebre fue la construcción de la Teoría de la Comunicación Constitutiva de las Organizaciones (CCO) con las iniciativas de Linda Putnam, en la Universidad de Santa Bárbara, y Robert McPhee, en la Universidad Estatal de Arizona. Desde luego, son fundamentales las aportaciones desde la Lingüística de la Universidad de Montreal en Canadá. Estos pensadores nos propusieron que en vez de asumir por dada la organización y luego analizar, señalan que la comunicación antecede a la organización, la constituye y los hace a través de flujos, intercambios, interacciones vivificantes, que le dan vida y la traen, por así decirlo, a la existencia.

En América Latina se ha desarrollado la Comunicación Organizacional de manera tan sofisticada debido a la complejidad de nuestra sociedad, a cuestiones que no únicamente dan seguimiento a asuntos gerenciales, de negocios, a retos de cómo "vender más", o "ser más productivos". Estamos ante organizaciones, instituciones públicas y organizaciones de la sociedad civil que cada vez nos demandan más retos para entender qué es lo que pasa al interior de estas. Para ello, hemos tenido que elaborar nuestros propios paradigmas teóricos y en muchas ocasiones estamos elaborando nuevas metodologías.

Margarida - Con relación a los temas que faltan, lo ideal sería que ya tuviéramos un mapeo de la producción de los diversos países. Pero yo considero que hoy hay temas que están en la agenda internacional, mundial, la cuestión que ya fue mencionada, la ética, la cuestión de la diversidad, la cuestión de género y yo diría la propia epistemología del campo. Por lo que acompañamos en los últimos años, diversos investigadores de países de la América Latina han demostrado mucho interés por los estudios de esas áreas de Comunicación Organizacional y Relaciones Públicas. Sobre todo Brasil, México y Colombia, por las investigaciones ya desarrolladas y por la literatura disponible en el mercado editorial.

En realidad, faltan más investigaciones colectivas y estudios comparativos de esas áreas por países para una mejor configuración de su historia y de su desarrollo en la región. La literatura disponible nos permite observar que los estudiosos latinoamericanos en muchos países ven la Comunicación Organizacional de una forma integral, involucrando las diversas modalidades/subáreas 
comunicacionales y valorándola cada vez más en términos de investigación académica y de prácticas. En el contexto de la realidad brasileña, los asuntos más tratados en el conjunto estudiado de disertaciones y tesis, que son generadas a partir del 2000, se caracterizan por una gran diversidad temática de objetos y mucha sintonía con la contemporaneidad y con las nuevas demandas de la sociedad y del mercado. Esto indica que el campo académico de la Comunicación Organizacional y de las Relaciones Públicas está atento a los acontecimientos y a las prácticas comunicativas en el contexto de las organizaciones y los procesos sociales.

Con bases en levantamientos y registros de la producción científica presentada en los centros de postgrado en Brasil, los temas más presentes en trabajos a nivel de maestría y doctorado, en las dos últimas décadas, son: la comunicación con la comunidad, las políticas públicas, la comunicación pública, la responsabilidad social, la comunicación en el tercer sector, la comunicación interna, el diálogo y las interacciones, la tecnología, las redes y los medios sociales, la comunicación y la sostenibilidad, la comunicación y la cultura organizativa, las narrativas y la memoria institucional de la comunicación, estrategias de comunicación, diversidad, comunicación organizacional y teoría de la complejidad (que tiene un énfasis grande, aquí está Cleusa Scroferneker, de la Pontificia Universidad Católica de Rio Grande do Sul), discurso organizacional, comunicación, mercado y marca (branding), y también evaluación y medición de resultados en Comunicación Organizacional y Relaciones Públicas. Del conjunto de tesis son los temas que están más presentes en el caso de Brasil.

Griselda - Margarida contestó unas de las siguientes preguntas en parte ¿cuáles son aquellos temas de comunicación organizacional explorados y cuáles nos hacen falta explorar? sobre todo con estos cambios de las organizaciones tan rápidos con las nuevas tecnologías que muchas veces nos rebasan.

María Antonieta - Yo hablaría de dos cosas básicamente. Una sería que nos hace falta entender mejor cuál sería la naturaleza misma de las organizaciones, entenderlas porque la comunicación constituye las organizaciones, es ese contexto es en donde ocurre la comunicación y de donde sale la comunicación a otras organizaciones. Y, bueno, yo voy a lanzar algo, una iniciativa, creo que de aquí de este tipo de reuniones debe salir un formato, más consensuado entre todos nosotros, para certificar al Comunicador Organizacional, creo que hace falta ese punto, que creo que deberíamos estar pensando seriamente en convertirnos, pues, en algo más que simplemente observadores, simplemente investigadores, de un fenómeno que tenemos y que ha sido tan interesante analizar. Y dar pasos más allá, donde podamos decir: ¿cuál es el conjunto de habilidades?, ¿cuál es el conjunto de saberes que tiene que reunir un comunicador organizacional para funcionar mejor en sociedad y dar un mejor servicio a las organizaciones?

Margarida - En nuestro grupo de aportes teóricos, se habló del tema: las grandes empresas y organizaciones tienen toda una estructura de comunicación, de inversiones, etcétera, pero es importante ver cómo podemos valorizar también las pequeñas organizaciones, porque las organizaciones sociales, los movimientos sociales y los grupos comunitarios también necesitan de estudios y metodologías. Los estudios deben privilegiar ese segmento de grupos minoritarios; la cuestión de género, los refugiados, son temas que están ahí y merecen ser investigados. Organizaciones pequeñas que trabajan con emprendimientos sociales es una realidad común que compartimos, verificar cómo estas organizaciones están planificando, gestionando sus procesos de comunicación. Es difícil responder esta pregunta sin tener un mapeo de la producción científica que es generada en los diversos países. Sin embargo, creo que temas como género, ética y cumplimiento, epistemología del propio campo todavía son poco explotados en América Latina. 
María Antonieta - Es una gran pregunta. Yo creo que es la pregunta central que nos tenemos que estar haciéndonos todos. Ciertamente, tenemos mucho que aportar para América Latina, en particular, en donde tenemos sociedades altamente desiguales, en donde son las pequeñas empresas, las micro que llevan la carga más fuerte de dar empleo a la sociedad y de producir los bienes y servicios que necesitamos. Considero que sí debemos pensar en estas problemáticas, en donde las desigualdades, no son solo socioeconómicas, sino también educativas, de género y la violencia, en donde también las organizaciones tienen mucho que hacer, porque a veces, en las escuelas como instituciones y en las organizaciones del trabajo en donde generamos estas actitudes de violencia y agresivas hacia las personas, hacia las mujeres y otros grupos minoritarios. Veo a América Latina como un campo muy basto con sus peculiaridades sociales que nos va a permitir ir viendo en dónde están aquellas necesidades más álgidas. Los invito a todos que cuando pensemos en Comunicación Organizacional, hay que dejar de pensar únicamente en las grandes empresas transnacionales como el lugar donde se da nuestra acción y en el lugar en donde ya están establecidas estas grandes unidades de comunicación o gabinetes. Nos debemos plantear la necesidad de incidir en otros tipos de organizaciones, como son las instituciones tanto las públicas como las privadas y las de gobierno. Debemos atender los retos que nos ponen los organismos internacionales, que también constituyen formas organizativas. Así mismo, queda por delante todo el espacio de las asociaciones del tercer sector: las filantropías, asociaciones civiles, instituciones de asistencia pública. En todas estas es la comunicación el proceso más importante de toda sociedad, de todo sistema, en toda unidad, incluidas las formas de vida biológicas. Sin comunicación no hay vida.

Y, bueno, desde luego ahora y siempre, invitamos a la discusión a los españoles, quienes por tantos años han contribuido en fundación y gestión de nuestros programas de formación doctoral y de maestría. Ellos han contribuido de manera importante a construir la disciplina, a reflexionar en conjunto, a observar los cambios y a sugerir nuevos caminos de andamiaje necesarios.

Margarida - Antonio Castillo y Ana Almansa ya son brasileñistas y latinistas, siempre presentes y con libros publicados en portugués también. Invertir en las investigaciones científicas con miras a mapear el "estado del arte" del campo en América Latina, y cómo se desarrolla la producción científica disponible en forma de tesis, libros, artículos en revistas científicas, congresos etc., puede ser un buen camino hacia posteriores estudios; realizar estudios comparativos del campo entre países y promover coloquios académicos como este, reuniendo investigadores y estudiosos para debatir las grandes demandas actuales de la sociedad latinoamericana, que, como bien lo señaló María Antonieta, es una sociedad totalmente desigual, con muchos problemas.

Muchas veces se destaca a Siria donde los refugiados están yendo para Europa, pero nosotros tenemos Venezuela, que tiene frontera con Colombia y con Brasil, son miles que salen, para Brasil ya más de 20 mil. Y ahora también de Nicaragua se están viniendo hacia Costa Rica. En el siglo XXI, los problemas de este tipo, de migraciones, personas en la frontera de Estados Unidos que se separan de sus hijos, son muchos problemas sociales, que los refugiados están buscando mejores condiciones de vida, son víctimas de una sociedad. Y todo esto es una realidad social crucial para nosotros. Debemos contemplar nuestros estudios de esas demandas. Necesitamos conocer y reflexionar sobre lo que estamos haciendo. Muchas veces evaluamos poco, y tenemos que ser más críticas también de lo que producimos.

Solo así vamos a avanzar y ocupar los debidos espacios en la comunidad académica internacional de las Ciencias de la Comunicación. Si realmente trabajamos con bases científicas como se habló, en general, los otros campos desconocen todos los avances, las teorías de las áreas, a veces nuestros propios pares de teorías de la comunicación de periodismo desconocen lo que desarrollamos en Comunicación Organizacional y Relaciones Públicas. Entonces, es una batalla para enfrentarse. Solo con la producción y el trabajo podemos ocupar más espacio. 
Griselda - Agradecemos a las doctoras María Antonieta y Margarida por esta entrevista que nos han permitido realizar, gracias por sus aportaciones en este espacio y en el campo.

Artículo recibido el 12.07.2019 y aprobado el 12.07.2019 